\title{
Risk factors and outcomes of contrast-induced nephropathy in hospitalised South Africans
}

\author{
J Banda, ${ }^{1,2}$ MB ChB, MMed; R Duarte, ${ }^{1}$ MSc, PhD; C Dickens, ${ }^{1}$ MSc, PhD; T Dix-Peek, ${ }^{1}$ MSc; M Muteba ${ }^{3}$ MB ChB, MSc; \\ G Paget, ${ }^{1,2}$ FCP, Cert Nephrol, MMed; V Mngomezulu, ${ }^{4}$ FRCRad; P Manga, ${ }^{1,5}$ FCP, FRCP, PhD; S Naicker, ${ }^{1,2}$ FCP, FRCP, PhD \\ ${ }^{1}$ Department of Internal Medicine, Faculty of Health Sciences, University of the Witwatersrand, Johannesburg, South Africa \\ ${ }^{2}$ Division of Nephrology, Charlotte Maxeke Johannesburg Academic Hospital, Johannesburg, South Africa \\ ${ }^{3}$ Health Sciences Research Office, University of the Witwatersrand, Johannesburg, South Africa \\ ${ }^{4}$ Division of Radiology, Charlotte Maxeke Johannesburg Academic Hospital, Johannesburg, South Africa \\ ${ }^{5}$ Division of Cardiology, Charlotte Maxeke Johannesburg Academic Hospital, Johannesburg, South Africa
}

Corresponding author: J Banda (katusib@yahoo.co.uk)

Background. Despite ranking third as a cause of hospital-acquired acute kidney injury (AKI), iatrogenic contrast-induced nephropathy (CIN) impacts significantly on morbidity and mortality and is associated with high hospital costs. In sub-Saharan Africa, the rates and risk factors for CIN and patient outcomes remain unexplored.

Methods. We conducted a prospective observational study at the Charlotte Maxeke Johannesburg Academic Hospital, South Africa, from 1 July 2014 to 30 July 2015. Hospitalised patients undergoing computed tomography scan contrast media administration and angiography were consecutively recruited to the study and followed up for development of AKI. CIN was defined as an increase in serum creatinine $>25 \%$ or an absolute increase of $>44 \mu \mathrm{mol} / \mathrm{L}$ from baseline at $48-72$ hours post exposure to contrast media. Outcome variables were the occurrence of CIN, length of hospitalisation and in-hospital mortality.

Results. We recruited 371 hospitalised patients with a mean (standard deviation) age of 49.3 (15.9). The rates of CIN, assessed using an absolute or relative increase in serum creatinine from baseline, were $4.6 \%$ and $16.4 \%$, respectively. Anaemia was an independent predictor for the development of CIN (risk ratio (RR) 1.71, 95\% confidence interval (CI) 1.01 - 2.87; $p=0.04$ ). The median serum albumin was $34 \mathrm{~g} / \mathrm{L}$ (interquartile range (IQR) 29 - 39.5) and $38 \mathrm{~g} / \mathrm{L}$ (IQR 31 - 42) in the CIN and control groups, respectively ( $p=0.01$ ), and showed a significant trend for CIN development (RR 1.68, 95\% CI 0.96 - 2.92; $p=0.06$ ). Mortality was significantly increased in the CIN group (22.4\% v. $6.8 \%$; $p<0.001)$, and CIN together with anaemia increased mortality twofold (RR $2.39,95 \%$ CI $1.20-4.75 ; p=0.01$ ) and threefold (RR $3.32,95 \%$ CI $1.48-7.43 ; p=0.003$ ), respectively. Conclusions. CIN has a relatively high incidence in sub-Saharan Africa and predicts poorer clinical outcomes. The presence of CIN and anaemia positively predicted mortality. Caution should be exercised in patients with hypoalbuminaemia and anaemia undergoing contrast media administration.

S Afr Med J 2016;106(7):699-703. DOI:10.7196/SAMJ.2016.v106i7.10429

Despite preventive guidelines, iatrogenic contrast-induced nephropathy (CIN) ranks third as a cause of hospital-acquired AKI, impacts significantly on morbidity and mortality, and is associated with longer stays in hospitals, with higher medical costs. ${ }^{[1-4]}$

The rates of CIN vary, depending on the study definitions employed and underlying risk factors, and range from $0.6 \%$ to $30 \%$ or higher among risk groups. ${ }^{[5-8]} \mathrm{CIN}$ is also associated with increased duration of hospitalisation and early and late mortality. In-hospital mortality due to CIN ranges from $7 \%$ to $22 \% \cdot{ }^{[9]}$ McCullough et al. ${ }^{[9]}$ reported early mortality of $1.1 \%$ in controls, $7.1 \%$ in CIN patients, and $35 \%$ in CIN patients that required dialysis. Owing to increased comorbidities, hospitalised patients have increased risk for developing CIN compared with ambulatory patients ${ }^{[10]}$

Inflammation and endothelial dysfunction, together with reactive oxygen species (ROS), are implicated in the pathogenesis of CIN. ${ }^{[8]}$ Iodinated contrast media directly injures the renal tubular epithelium by producing ROS radicals that cause intra-renal vasoconstriction leading to ischaemia and death of tubular cells. ${ }^{[8,10,11]}$ Serum albumin is an important antioxidant that reduces the formation of oxygen free radicals and is important in expanding intravascular volume. ${ }^{[12,13]}$ However, the role of serum albumin in reducing the incidence of $\mathrm{CIN}$ remains unexplored.

Sub-Saharan Africa has a dearth of data on rates of CIN. This study investigated the rates of CIN together with the influence of serum albumin, albuminuria, age, haemoglobin, and glomerular filtration rate (GFR) levels on CIN and patient outcomes.

\section{Methods}

Study design, setting and population

This study was a prospective observational study conducted at Charlotte Maxeke Johannesburg Academic Hospital, Johannesburg, South Africa, from 1 July 2014 to 30 July 2015. Ethical approval was obtained from the human research ethics committee (HREC) of the University of the Witwatersrand and informed written consent was obtained from all patients. We consecutively recruited hospitalised patients undergoing computed tomography (CT) and angiography from the Divisions of Radiology and Cardiology, respectively. The following exclusion criteria were used: $<18$ years of age; evidence of pre-existing AKI (clinical or laboratory); end-stage renal disease (ESRD); renal replacement therapy (RRT); prior contrast media administration in the preceding 7 days; pregnancy; or incomplete data.

\section{Study procedures}

The study physician reviewed participants' medical records and examined the participants in order to determine pre-existing risk factors for CIN. The medical records were reviewed to determine patient duration of hospitalisation, dialysis requirements and mortality. Study phlebotomists collected blood samples prior to and post contrast media administration. Pre contrast, urine was collected and analysed for microalbuminuria using the Chemistrip Micral 30 immune assay test (Roche 11544039172). Serum creatinine was analysed using the Jaffe method (Siemens ADVIA 1200 chemistry 
system) and estimated GFR (eGFR) was calculated using the four variable CKD-EPI equation. ${ }^{[14]}$ Iopamidol (Jopamiron 370, Axim Pharmaceuticals, Italy), a low osmolar contrast agent, was used in all CT scan and angiography procedures.

\section{Study outcome measures}

The primary endpoint was the occurrence of CIN, defined as a serum creatinine increase of $>25 \%$ from baseline or an absolute increase of $44 \mu \mathrm{mol} / \mathrm{L}$ assessed within $48-72$ hours post contrast media administration as per the 2011 updated European Society of Urogenital Radiology (ESUR) guidelines. ${ }^{[15-17]}$ Several studies have used the ESUR definition.
The secondary endpoints were death and duration of hospitalisation obtained from patient records. The CIN group was reviewed for renal recovery and progression to chronic kidney disease (CKD) at 3 months following the contrast exposure.

\section{Statistical analysis}

Statistical analyses were performed using Stata version 13 software (Stata Inc., USA).

Using conservative estimates for the prevalence of CIN, a minimum sample size of 323 was required to sufficiently power this study. Continuous variables are presented as means and standard deviations

Table 1. Baseline characteristics for study participants

\begin{tabular}{|c|c|c|c|c|}
\hline Characteristic $^{*}$ & All $(N=371)$ & $\mathrm{CIN}-(n=310)$ & $\mathrm{CIN}+(n=310)$ & $p$-value \\
\hline Age (years), mean (SD) & $49.3(15.9)$ & $48.7(16.3)$ & $52.1(14.1)$ & 0.11 \\
\hline Gender (male), $n(\%)$ & $210(43.4)$ & $174(56.1)$ & $36(59.0)$ & 0.86 \\
\hline Diabetes mellitus, $n(\%)$ & $48(13.2)$ & $42(13.8)$ & $6(10.3)$ & 0.48 \\
\hline HTN, $n(\%)$ & $93(25.8)$ & $78(25.7)$ & $15(25.9$ & 0.98 \\
\hline Malignancy, $n(\%)$ & $159(42.9)$ & $136(43.9)$ & $23(37.8)$ & 0.34 \\
\hline Cardiac disease, $n(\%)$ & $56(15.1)$ & $45(14.5)$ & $11(18.0)$ & 0.48 \\
\hline Stable CKD, $n(\%)$ & $13(3.6)$ & $9(2.9)$ & $4(6.9)$ & 0.13 \\
\hline Systolic BP (mmHg), median (IQR) & $119(109-130)$ & $119(109.5-130)$ & $120(106-132)$ & 0.43 \\
\hline Diastolic BP (mmHg), median (IQR) & $73(66-83)$ & $73(65-82.5)$ & $74(68-84)$ & 0.18 \\
\hline Hospitalisation (days), median (IQR) & $11(6-20)$ & $11(5-18)$ & $15(8-23)$ & 0.52 \\
\hline Mortality, $n(\%)$ & $33(9.3)$ & $20(6.8)$ & $13(22.4)$ & $<0.001$ \\
\hline Prehydration, $n(\%)$ & $38(17.7)$ & $28(15.8)$ & $10(26.3)$ & 0.12 \\
\hline $\mathrm{N}$ acetyl cysteine, $n(\%)$ & $3(0.8)$ & $3(1.0)$ & $0(0.0)$ & 0.45 \\
\hline ACEI/ARBs, $n(\%)$ & $69(19.2)$ & $59(19.5)$ & $10(17.5)$ & 0.73 \\
\hline Statins, $n(\%)$ & $67(18.6)$ & $55(18.2)$ & $12(20.7)$ & 0.66 \\
\hline Diuretics, $n(\%)$ & $70(19.5)$ & $55(18.3)$ & $15(25.9)$ & 0.18 \\
\hline Serum creatinine $(\mu \mathrm{mol} / \mathrm{L})$ & $68(53-85)$ & $68.5(54-84)$ & $61(52-89)$ & 0.49 \\
\hline eGFR $\left(\mathrm{mL} / \mathrm{min} / 1.73 \mathrm{~m}^{2}\right)$, median (IQR) & $110.8(89.5-134)$ & $113.4(89.9-134)$ & $107(89.5-133)$ & 0.84 \\
\hline $\left.\mathrm{eGFR}<60 \mathrm{~mL} / \mathrm{min} / 1.73 \mathrm{~m}^{2}\right), n(\%)$ & $13(3.5)$ & $10(76.9)$ & $3(23.1)$ & 0.43 \\
\hline Urea (mmol/L), median (IQR) & $4.8(3.6-6.3)$ & $4.9(3.5-6.3)$ & $4.7(4-6.7)$ & 0.08 \\
\hline Microalbuminuria, $n(\%)$ & $58(38.1)$ & $46(36.8)$ & $12(44.4)$ & 0.46 \\
\hline Serum albumin $(\mathrm{g} / \mathrm{L})$, median (IQR) & $37(30-41)$ & $38(31-42)$ & $34(29-39.5)$ & 0.01 \\
\hline Serum albumin $<35 \mathrm{~g} / \mathrm{L}, n(\%)$ & $113(41.0)$ & $82(37.4)$ & $31(55.4)$ & 0.02 \\
\hline $\mathrm{Hb}(\mathrm{g} / \mathrm{dL})$, median (IQR) & $12.3(9.8-14.3)$ & $12.5(10.1-14.4)$ & $11(9.3-13.4)$ & 0.01 \\
\hline Anaemia $^{\dagger}(\mathrm{Hb}<11 \mathrm{~g} / \mathrm{dL}), n(\%)$ & $117(34.5)$ & $89(32.1)$ & $28(50.9)$ & 0.01 \\
\hline White cell count $\left(\times 10^{9} / \mathrm{L}\right)$, median (IQR) & $8.1(6.3-11.7)$ & $7.9(6.3-11.7)$ & $8.6(5.9-13.9)$ & 0.70 \\
\hline CRP (mg/L), median (IQR) & $25.5(10-70)$ & $25.5(10-70)$ & $55(12-115)$ & 0.15 \\
\hline HIV-positive, $n(\%)$ & $74(36.8)$ & $61(37.2)$ & $13(35.1)$ & 0.58 \\
\hline Sodium (mmol/L), median (IQR) & $139(135-141)$ & $139(136-141)$ & $138(135-140)$ & 0.17 \\
\hline Bicarbonate (mmol/L), median (IQR) & $25(22-27)$ & $25(22-27)$ & $24(20-26)$ & 0.45 \\
\hline Phosphate (mmol/L), median (IQR) & $1.1(0.90-1.2)$ & $1.10(0.90-1.24)$ & $1.00(0.81-1.20)$ & 0.10 \\
\hline Contrast volume (mL), mean (SD) & $103.8(39.9)$ & $103.7(37.3)$ & $104.2(51.8)$ & 0.95 \\
\hline Contrast dose:CrCL ratio & $0.88(0.72-1.16)$ & $0.87(0.72-1.17)$ & $0.94(0.71-1.1)$ & 0.31 \\
\hline Ejection fraction $\%$, mean (SD) & $63.1(14.0)$ & $63.1(14.0)$ & $59.1(13.3)$ & 0.31 \\
\hline
\end{tabular}




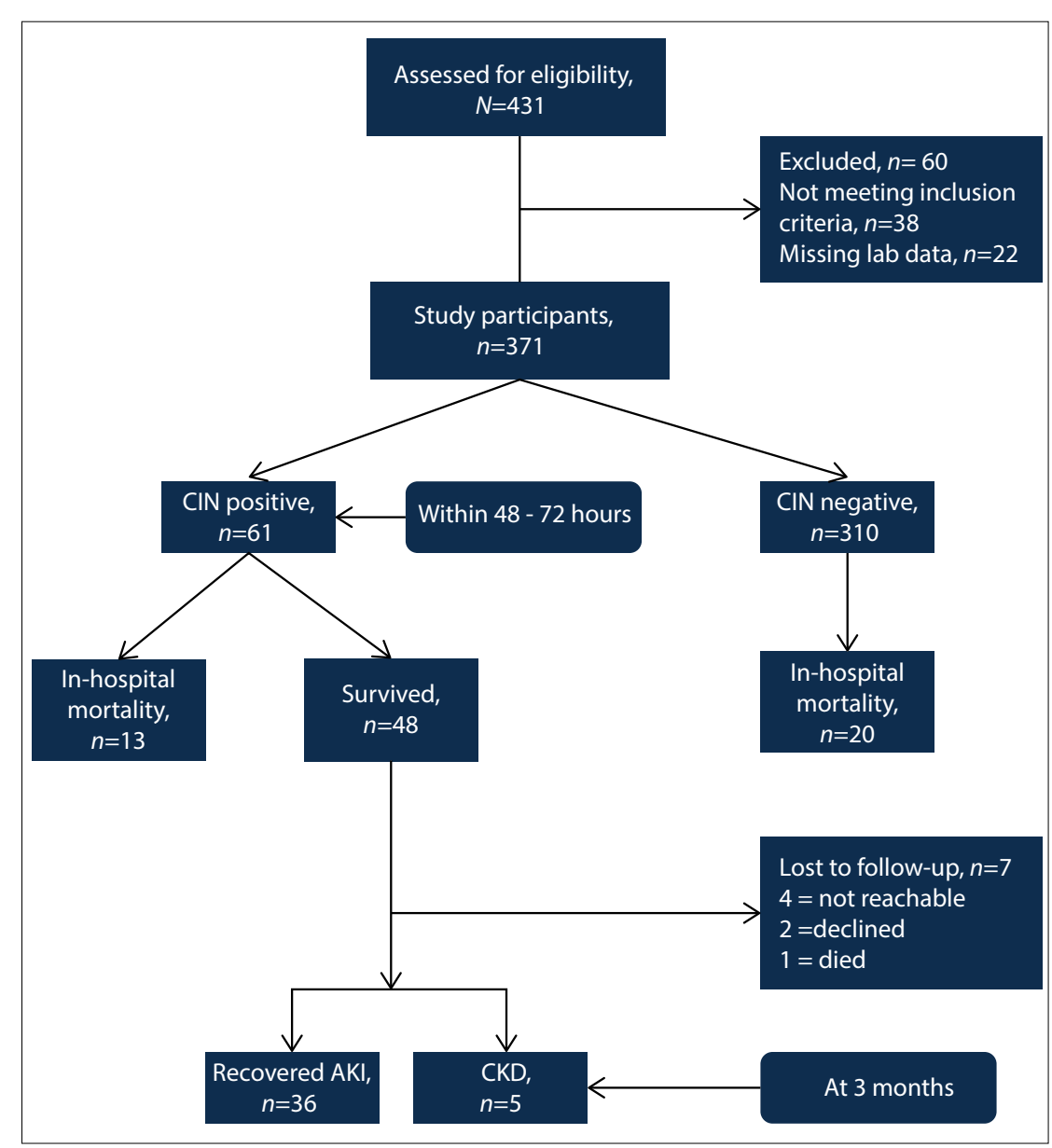

Fig. 1. Flow chart of study participants.

Table 2. Independent predictors for CIN

\begin{tabular}{|c|c|c|c|c|c|c|}
\hline & \multicolumn{3}{|c|}{ Unadjusted } & \multicolumn{3}{|c|}{ Adjusted } \\
\hline & $\mathbf{R R}$ & $95 \% \mathrm{CI}$ & $p$-value & $\mathbf{R R}$ & $95 \% \mathrm{CI}$ & $p$-value \\
\hline Age & 1.01 & $0.99-1.02$ & 0.14 & 1.00 & $0.99-1.03$ & 0.89 \\
\hline Gender & 1.04 & $0.66-1.66$ & 0.86 & 1.37 & $0.82-2.29$ & 0.22 \\
\hline $\begin{array}{l}\text { Albumin level } \\
(<35 \mathrm{~g} / \mathrm{dL})\end{array}$ & 1.60 & $1.00-2.56$ & 0.05 & 1.68 & $0.96-2.92$ & 0.06 \\
\hline Anaemia & 0.89 & $0.81-0.98$ & 0.025 & 1.71 & $1.01-2.87$ & 0.04 \\
\hline Baseline eGFR & 0.99 & $0.98-1.00$ & 0.70 & & & \\
\hline
\end{tabular}

\section{Table 3. Independent predictors for mortality}

\begin{tabular}{lllllll}
\hline & \multicolumn{3}{c}{ Unadjusted } & \multicolumn{3}{c}{ Adjusted } \\
\cline { 2 - 7 } & RR & $\mathbf{9 5 \%}$ CI & $\boldsymbol{p}$-value & RR & $\mathbf{9 5 \% ~ C I ~}$ & $\boldsymbol{p}$-value \\
\hline CIN & 3.27 & $1.72-6.18$ & $<0.0001$ & 2.39 & $1.20-4.75$ & $0.01^{*}$ \\
Age & 1.00 & $0.96-1.02$ & 0.98 & 0.99 & $0.98-1.01$ & 0.91 \\
Anaemia & 3.61 & $1.75-7.45$ & 0.001 & 3.32 & $1.48-7.43$ & $0.003^{*}$ \\
Contrast medium dose & 0.99 & $0.98-0.99$ & 0.02 & 0.99 & $0.99-1.00$ & 0.55 \\
Gender & 1.06 & $0.55-2.05$ & 0.18 & 0.96 & $0.49-1.89$ & 0.91 \\
*Included in final model were age, gender, anaemia, contrast media volume and CIN. & & &
\end{tabular}

when normally distributed, and as medians and interquartile ranges (IQRs) when nonnormally distributed. Categorical variables are presented as counts and frequencies. Comparisons between $\mathrm{CIN}$-positive (CIN+) and $\mathrm{CIN}$-negative (CIN-) groups were assessed using a $t$-test for normally distributed continuous variables, a Wilcoxon rank sum test for non-normally distributed continuous variables and a Pearson $\chi^{2}$ test for categorical variables. $p$-values of $<0.05$ were considered significant. Multivariate analyses examined the associations of CIN and mortality with various risk factors and a generalised linear regression model built to adjust for confounding effects.

\section{Results}

\section{Baseline characteristics}

Participants' baseline characteristics are reported in Table 1. After excluding 60 noneligible patients (Fig. 1), 371 participants remained in the study; $74.9 \%$ were black, $18.9 \%$ white, $3.8 \%$ Indian and $2.4 \%$ of mixed race. Ages ranged from 18 to 92 years (mean (standard deviation) 49.3 (15.9)). The rate of CIN was $16.4 \%$ (61/371) when based on a $25 \%$ increase from baseline in serum creatinine and $4.6 \%$ when based on an absolute increase of $>44 \mu \mathrm{mol} / \mathrm{L}$. Baseline serum creatinine and GFR were not significantly different between the CIN and control groups; almost $97 \%$ had baseline GFR $\geq 60 \mathrm{~mL} / \mathrm{min} / 1.73 \mathrm{~m}^{2}$, while the remaining $3 \%$ had baseline GFR ranging from 42 to $59 \mathrm{~mL} / \mathrm{min} / 1.73 \mathrm{~m}^{2}$. Compared with the CIN-free group, CIN patients had significantly lower levels of baseline serum albumin and haemoglobin.

\section{Independent predictors of CIN}

After adjusting for all possible confounders, anaemia was found to be a predictor for CIN. Also, low serum albumin was positively associated with CIN development (Table 2).

\section{CIN and mortality}

Both duration of hospitalisation and mortality were higher in the CIN group but only mortality was significantly increased $(p<0.001)$ (Fig. 2). None of the patients who developed CIN required dialysis. Nonsignificant variables that included cardiac disease, malignancy, contrast volume and baseline eGFR and albumin were excluded in the final model. In multivariable analysis CIN and anaemia were positive predictors for mortality (Table 3 ).

\section{Discussion}

The present study demonstrated a relatively high rate of CIN in hospitalised patients. 


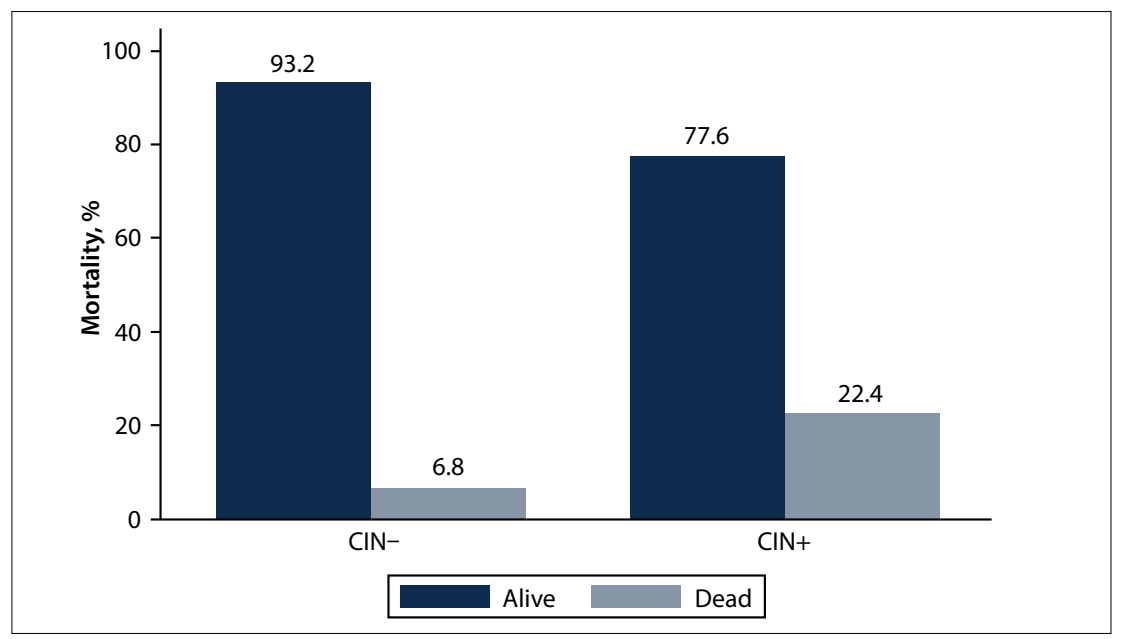

Fig. 2. Contrast-induced nephropathy and mortality $(C I N-v$. CIN+, $\mathrm{p}<0.001)$.

Furthermore, the development of CIN resulted in increased mortality and highlighted the association of CIN with poorer clinical outcomes.

Our study showed a $16.4 \%$ rate of $\mathrm{CIN}$, which was slightly higher than reported in most recent studies. The reported incidence of CIN is variable, depending on the study definitions employed and underlying risk factors, and ranges from $5 \%$ to $30 \%$ overall. ${ }^{[5-8,18,19]}$ Recent observational studies have reported low rates of CIN among hospitalised patients. ${ }^{[20,21]}$ ShamsEddin Taher ${ }^{[21]}$ observed a $11.5 \%$ rate of CIN in patients undergoing percutaneous intervention (PCI) while Selistre et al. ${ }^{[20]}$ reported a $14 \%$ incidence of CIN in patients undergoing contrast CT administration in Brazil. A randomised controlled trial (RCT) in the USA found a $16.1 \%$ incidence of CIN in patients undergoing PCI. ${ }^{[22]}$ The heterogeneous study population, variable comorbidities and non-standardised preventive prescriptions used by clinicians may explain the high rate of CIN in our study.

In this study, CIN patients had significantly lower levels of precontrast serum albumin compared with controls and showed a significant positive trend towards the development of CIN with serum albumin levels. Serum albumin is an important renoprotective, anti-inflammatory and antioxidant agent acting via several mechanisms; serum albumin binds non-ceruloplasmin copper and iron, which are important in the formation of ROS, and maintains renal perfusion by dilating renal vessels via its binding to nitrogen oxide, forming S-nitro-albumin. ${ }^{[12,23-26]}$ However, during inflammation, albumin synthesis is reduced and activated neutrophils increase the levels of hypochlorous acid, which inactivates albumin. ${ }^{[12,13,23]}$ Low serum albumin is probably a biomarker reflecting baseline susceptibility to acute or chronic inflammation or undernutrition. In two previous studies, Murat et al. ${ }^{[23]}$ and Song et al. ${ }^{[24]}$ found a positive association between low serum albumin and an increased risk for the development of CIN. A meta-analysis showed a positive association between decreased serum albumin and development of AKI, as well as increased morbidity and mortality. ${ }^{[27]}$ The odds of developing AKI doubled with reduced serum albumin. ${ }^{[26]}$ Lee et al. ${ }^{[28]}$ reported twofold odds of developing postoperative AKI with decreased serum albumin.

Of interest in our study was the impact of baseline anaemia on patient outcomes. The rate of baseline anaemia was relatively high in our study population compared with controls, and was associated with development of CIN and a threefold mortality risk. Limited studies have explored the association of anaemia with CIN and patient outcomes. Li et al. ${ }^{[26]}$ and Chong et al. ${ }^{[29]}$ in observational studies reported a positive association between baseline anaemia and CIN In these studies the presence of anaemia was associated with an almost twofold risk of developing CIN. ${ }^{[26,29]}$ In patients undergoing PCI, Mckechnie et al. ${ }^{[30]}$ similarly reported baseline anaemia as an independent predictor for mortality. The increased risk of CIN in patients with anaemia and poorer clinical outcomes is multifactorial in aetiology; in the presence of anaemia, CIN-induced renal ischaemia and hypoxia are significantly worsened in the outer renal medulla amid already prevailing low oxygen tension, and oxygen affinity and demand are increased. ${ }^{[26,31,32]}$ Anaemia also signifies underlying comorbidities; therefore it is a biomarker for severity of disease at the time of contrast media administration, and increased mortality risk. ${ }^{[26,30]}$
Age, baseline renal function (determined by eGFR) and prehydration were not associated with the prevention of CIN in our study. The positive association between CIN and age has been shown in the age group above 62 years. ${ }^{[11]}$ An RCT found age above 75 years together with diabetes mellitus to be a predictor for CIN. ${ }^{[22]}$ In younger type 2 diabetic patients undergoing PCI, Zaki et al. ${ }^{[25]}$ reported age above 57 years to be a positive predictor for CIN. Our study population was relatively young, and fewer of our patients had multivessel disease compared with previous studies. Baseline eGFR level was insignificantly associated with CIN in our study cohort. Previous studies have shown decreased risk of developing CIN with eGFR $>60 \mathrm{~mL} /$ $\mathrm{min} / 1.73 \mathrm{~m}^{2} \cdot{ }^{[20]} \mathrm{In}$ an observational study, Weisbord et al. ${ }^{[33]}$ found $<1 \%$ incidence of CIN in patients with eGFR $>45-60 \mathrm{~mL} /$ $\mathrm{min} / 1.73 \mathrm{~m}^{2}$ after contrast administration. In support of our study, Chong et al. ${ }^{[29]}$ found insignificant association between normal renal status and CIN development. In a cohort study of $80 \%$ study participants with eGFR $>60 \mathrm{~mL} / \mathrm{min} / 1.73 \mathrm{~m}^{2}$, Selistre et al. ${ }^{[20]}$ also reported no association between baseline eGFR and risk for CIN. In our study cohort, $97 \%$ of study participants had normal baseline renal function based on eGFR, with the remaining having eGFR ranging from 42 to $59 \mathrm{~mL} / \mathrm{min} / 1.73 \mathrm{~m}^{2}$ with no significant association with CIN.

Prehydration therapy was used in only $18 \%$ of patients in our study cohort compared with $70 \%$ seen in developed countries, ${ }^{[34]}$ and prehydration showed no impact on reducing the incidence of CIN. It is possible that only the patients at highest risk for CIN received prehydration and this possibly obscured any beneficial effects. In previous studies, Selistre et al. ${ }^{[20]}$ observed no association between fluid administration and CIN occurrence. Diogo et al. ${ }^{[35]}$ reported a low rate of prehydration therapy in Brazil.

Similar to previous findings, our study demonstrated a higher mortality in the CIN group compared with controls, and CIN and anaemia were independent predictors of mortality. ${ }^{\left[{ }^{[6]}\right.}$ Rihal et al. ${ }^{[37]}$ in a retrospective registry review at the Mayo Clinic, reported in-hospital mortality rates of $22.0 \%$ and $1.4 \%$ in the CIN and controls groups, respectively, and CIN was positively associated with a 10 -fold increased risk of death. Furthermore, cardiac disease was associated with a twofold increased risk of death. Sadeghi et al. ${ }^{[36,38]}$ in a multicentre study found that 30-day mortality was significantly higher among patients with CIN compared with controls $(16.2 \%$ v. $1.2 \%$; $p<0.001)$. In-hospital mor- 
tality due to CIN in the National Cardiovascular Registry in USA was reported as $9.1 \%{ }^{[39]}$

Development of CIN also reflects underlying disease comorbidities at the time of contrast media administration. Despite increased mortality in the CIN group compared with controls, Sadeghi et $a l .{ }^{[38]}$ found underlying cardiac disease as a positive predictor for mortality. In other studies, age, cardiac disease and the requirement of dialysis were predictors for mortality in CIN participants. ${ }^{\left[{ }^{[6]}\right.}$ In our study, among the 13 deaths in the CIN group, 7 had a diagnosis of malignancy; however, after adjusting for possible confounders, only CIN and anaemia (and not malignancy) were predictors of mortality.

The strength of our study is that it was a prospective cohort with a large sample size. However, it is limited by being a single-centre, non-interventional study conducted at a tertiary hospital. The study consisted of a heterogeneous population, and prevention guidelines for CIN were not standardised or uniformly implemented.

\section{Conclusion}

Our study highlights the high rates of CIN and associated poorer clinical outcomes. Caution should be used when administering iodinated contrast media to patients with hypoalbuminaemia and anaemia. To our knowledge, this is the first prospective study examining the risk and outcomes of CIN in sub-Saharan Africa. This study advocates that preventive treatment guidelines for CIN should be highlighted and standardised across various hospital departments.

Acknowledgements. This study was supported by Carnegie Corporation of New York grant B 8749.R01 and has been made possible through Dr Justor Banda's International Society of Nephrology (ISN) fellowship training at the University of the Witwatersrand. The authors also acknowledge Dr Ben Andrews (Memphis, TN, USA) for technical advice.

\section{References}

1. Shema L, Ore L, Geron R, Kristal B. Contrast-induced nephropathy among Israeli hospitalized patients: Incidence, risk factors, length of stay and mortality. Isr Med Assoc J 2009;11(8):460-464.

patients: Incidence, risk factors, length of stay and mortality. Isr Med Assoc J 2009;11(8):460-464.
2. Evola S, Lunetta M, Macaione F, et al. Risk factors for contrast induced nephropathy: A study among Evola S, Lunetta M, Macaione F, et al. Risk factors for contrast induce
Italian patients. Indian Heart J 2012;64(5):484-491. DOI:10.1016/j.ihj

3. Mitchell AM, Jones AE, Tumlin JA, Kline JA. 1-year mortality following contrast-induced nephropathy. Am J Intern Med 2103;1(1):1-6. DOI: 10.11648/j.ajim

4. Mitchell AM, Jones AE, Tumlin JA, Kline JA. Incidence of contrast-induced nephropathy after contrastenhanced computed tomography in the outpatient setting. Clin J Am Soc Nephrol 201;5(1):4-9. DOI:10.2215/CJN.05200709

5. Lameire NH. Contrast induced nephropathy: Prevention and risk reduction. Nephrol Dial Transplant 2006;21(6):i11-2.

6. Finn WF. The clinical and renal consequences of contrast induced nephropathy. Nephrol Dial Transplant 2006;21(6): i2-10.

7. Deek H, Newton P, Sheerin N, Davison PM. Contrast media induced nephropathy: A literature review of the available evidence and recommendations for practice. Aust Crit Care 2014;27(4):166-171. DOI:10.1016/j.aucc

8. Pisani A, Riccio E, Andreucci M, et al. Role of reactive oxygen species in pathogenesis of radio contrastinduced nephropathy. BioMed Res Int 2013 (2013), Article ID 868321:1-6. DOI:10.1155/2013/868321

9. McCullough PA, Woyln R, Rochner LL, Levin RN, O'Neill WW. Acute renal failure after coronary intervention: Incidence, risk factors and relationship to mortality. Am J Med 1997;103(5):368-375.

10. Meinel FG, de Cecco CN, Schoepf UJ, Katzberg R. Contrast-induced acute kidney injury: Definition, epidemiology, and outcome. BioMed Res Int 2014 (2014), Article ID 859328:1-6. DOI:10.1155/2014/859328 11. Chao CT. Epidemiology, clinical features and diagnosis of contrast induced nephropathy: A brief review. Gen Med 2013;1(1):1-5. DOI:10.4172/2327-5146.1000102
12. Halliwell B. Albumin: An important extracellular antioxidant? Biochem Pharmacol 1988;37(4):569-571.

13. Taverna M, Marie AL, Mira JP, Guidet B. Specific antioxidant properties of human serum albumin Ann Intensive Care 2013;3(4):1-7. DOI:10.1186/2110-5820-3-4

14. Levey AS, Stevens LA, Schmid CH, et al. A new equation to estimate glomerular filtration rate. Ann Levey AS, Stevens LA, Schmid CH, et al. A new equation to estimate glomerular
Intern Med 2009;150(9):604-612. DOI:10.7326/0003-4819-150-9-200905050-00006

15. Bellin MF, Jakobsen JA, Tomassin I, et al. Contrast medium extravasation injury: Guidelines for prevention and management. Eur Radiol 2002;12(11):2807-2812.
pellin

prevention and management. Eur Radiol 2002;12(11):2807-2812.
16. Thomsen HS, Morcos SK. Contrast media and the kidney: European Society of Urogenital Radiology 6. Thomsen HS, Morcos SK. Contrast media and the kidney: European Society of Ur
(ESUR) guidelines. Br J Radiol 2003;76(908):513-518. DOI:10.1259/bjr/26964464

17. Mehran R, Nikolsky E. Contrast-induced nephropathy: Definition, epidemiology, and patients at risk Kidney Int Suppl 2006;100:S11-S15.

18. Golshahi J, Nasri H, Gharipour M. Contrast induced nephropathy: A literature review. J Nephropathol 2014;3(2):51-56. DOI:10.12860/jnp.2014.12

19. Neyra JA, Shah S, Mooney R, et al. Contrast-induced acute kidney injury following coronary angiography: A cohort study of hospitalized patients with or without chronic kidney disease. Nephrol Dial Transplant 2013;28:1463-1467. DOI:10.1093/ndt/gft082

20. Selistre LD, Souza VC, Dubourg L, et al. Contrast induced nephropathy after computer tomography. J Bras Nefrol 2015;37(1):27-31. DOI:10.5935/0101-2800.20150005

21. Shams-Eddin Taher H, Hassan AKM, Dimitry SR, Mahfouz AK. Predicting contrast induced nephropathy post coronary intervention: A prospective cohort study. Egypt Heart J 2015;67(4):337343. DOI:10.1016/i.ehj.2015.02.001

22. Narula A, Mehran R, Weisz G, et al. Contrast induced acute kidney injury after primary coronary intervention: Results from the HORIZONS-AMI sub study. Eur Heart J 2014;35:1533-1540. DOI:10.1093/eurheartj/ehu063

23. Murat SN, Kurtul A, Yarlioglues M. Impact of serum albumin levels on contrast induced acute kidney injury in patients with acute coronary syndromes treated with percutaneous coronary intervention Angiology 2015;66:1-8. DOI:10.1177/0003319714551979

24. Song JS, Kim SI, Kim W, et al. Incidence and risk factors of contrast-induced nephropathy after bronchial arteriography or bronchial artery embolization. Tuberc Respir Dis 2013;74:163-168. DOI:10.4046/trd.2013.74.4.163

25. Zaki T, Samir A, Mohammed T, Galal H, Samir W. Assessment of estimated GFR and clinical predictor of contrast induced nephropathy among diabetic patients undergoing cardiac catheterization. Egypt Heart J 2015;67:249-258.DOI:10.1016/j.ehj.2014.07.001

26. Li WH, Li DY, Han F, et al. Impact of anemia on contrast-induced nephropathy (CIN) in patients undergoing percutaneous coronary interventions. Int Urol Nephrol 2013;45:1065-1070. DOI:10.1016/j. undergoing perc

27. Wiedermann CJ, Wiedermann W, Joannisdis M. Hypoalbuminemia and acute kidney injury: A metaanalysis of observational clinical studies. Intensive Care Med 2010;36:1657-1665. DOI:10.1007/s00134010-1928- 2

28. Lee $\mathrm{EH}, \mathrm{Baek} \mathrm{SH}$, Chin JH, et al. Pre-operative hypoalbuminemia is a major risk factor for acute kidney injury following off-pump coronary artery bypass surgery. Intensive Care Med 2012;38:1478-1486. DOI:10.1007/s00134-012-2599-8

29. Chong E, Poh K, Liang S, Tan HC. Risk factors and clinical outcomes for contrast-induced nephropathy after percutaneous coronary intervention in patients with normal serum creatinine. Ann Acad Med Singapore 2010;39(5):374-380.

30. McKechnie RS, Smith D, Montoye C, et al. Prognostic implication of anemia on in-hospital outcomes after percutaneous coronary intervention. Circulation 2004;110:271-277. DOI:10.1161/01. CIR.0000134964.01697.C7

31. Shacham Y, Gal-Oz A, Leshem-Rubinow E, et al. Association of admission hemoglobin levels and acute kidney injury among myocardial infarction patients treated with primary percutaneous intervention. Can J Cardiol 2015;31(1):50-55. DOI:10.1016/j.cica. 2014.11.001

32. Murakami R, Kumita S, Hayashi $\mathrm{H}$, et al. Anemia and the risk of contrast-induced nephropathy Murakami R, Kumita S, Hayashi H, et al. Anemia and the risk of contrast-induced nephropathy
in patients with renal insufficiency undergoing contrast-enhanced MDCT. Eur I Radiol 2013; 82(10):e521-e524. DOI:10.1016/j.ejrad.2013.06.004

33. Weisbord SD, Mor MK, Resnick AL, Hartwig KC. Incidence and outcomes of contrast-induced AKI following computed tomography. Clin J Am Soc Nephrol 2008;3:1274-1281. DOI:10.2215/CJN.01260308

34. Schilp J, de Blok C, Langelaan M, et al. Guideline adherence for identification and hydration of high-risk hospital patients for contrast-induced nephropathy. BMC Nephrol 2014;15:1471-2369. DOI:10.1186/1471-2369-15-2

35. Diogo LP, Bahlis LF, Carvalhal GF. Computerized tomography contrast induced nephropathy (CIN) among adult inpatients. J Bras Nefrol 2014;36:446-450. DOI:10.5935/0101-2800.20140064

36. Rudnick M, Feldman H. Contrast induced nephropathy: What are the true clinical consequences? Clin J Am Soc Nephrol 2008:3(1):263-272. DOI:10.2215/CIN.03690907

37. Rihal CS, Textor SC, Grill DE, et al. Incidence and prognostic importance of acute renal failure after percutaneous coronary intervention. Circulation 2002;105:2259-2264. DOI:10.1161/01. after percutaneous cor

38. Sadeghi HM, Stone GW, Grines CL, et al. Impact of renal insufficiency in patients undergoing primary angioplasty for acute myocardial infarction. Circulation 2003;108:2769-2775. DOI:10.1161/01. CIR.0000103623.63687.21

39. Tsai TT, Patel UD, Chang TI, et al. Contemporary incidence, predictors, and outcomes of acute kidney injury in patients undergoing percutaneous coronary interventions: Insights from the NCDR CathPCI registry. JACC Cardiovasc Interv 2014;7:1-9. DOI:10.1016/j.jcin.2013.06.016

Accepted 20 February 2016 